\title{
cAMP pulsing of denuded mouse oocytes increases meiotic resumption via activation of $\mathrm{AMP}$-activated protein kinase
}

\author{
Jing Chen, Maggie M Chi ${ }^{1}$, Kelle H Moley ${ }^{1}$ and Stephen M Downs \\ Department of Biological Sciences, Marquette University, Milwaukee, Wisconsin 53233, USA and ${ }^{1}$ Department \\ of Obstetrics and Gynecology, Washington University School of Medicine, Saint Louis, Missouri 63110, USA
}

Correspondence should be addressed to S M Downs; Email: stephen.downs@marquette.edu

\begin{abstract}
cAMP plays a critical role in the control of oocyte maturation, as a high level of cAMP maintains oocyte arrest at the first meiotic prophase. Yet this study shows that pulsing meiotically arrested denuded oocytes (DO) with cAMP induces oocyte maturation through the activation of AMP-activated protein kinase (PRKA). Short-term (3 h) pulsing of meiotically arrested oocytes with forskolin, an adenyl cyclase (AC) activator, increased oocyte cAMP, led to elevated AMP, and induced oocyte meiotic resumption compared to oocytes continuously cultured in the control medium with or without forskolin. Western analysis showed that germinal vesicle (GV)-stage oocytes after forskolin pulsing contained increased levels of phospho-acetyl CoA carboxylase (pACACA), a primary substrate of PRKA. Pulsing oocytes with the phosphodiesterase (PDE)-sensitive cAMP analog, 8-bromo-cAMP (8-Br-cAMP), also increased pACACA and pPRKA levels in GV-stage oocytes and induced oocyte meiotic resumption. Moreover, the PRKA inhibitors, compound C and araA, prevented 8-Br-cAMP pulsing-induced maturation. The lack of effect on meiotic induction and PRKA activation when oocytes were pulsed with the PDE-resistant activators of cAMP-dependent protein kinase, Sp-cAMP-AM and Sp-5,6-DCI-cBIMPS, suggests that cAMP degradation is required for pulsing-induced maturation. Pulsing oocytes with the exchange protein directly activated by cAMP (Epac)-specific activator, 8-CPT-2'-O-Me-cAMP, had no stimulatory effect on oocyte maturation, suggesting Epac is not involved in the pulsing-induced maturation. Taken together, these data support the idea that a transient increase in oocyte cAMP can induce meiotic resumption via activation of PRKA.

Reproduction (2009) 138 759-770
\end{abstract}

\section{Introduction}

Fully-grown mammalian oocytes within healthy preovulatory follicles are arrested in the first meiotic prophase until the gonadotropins surge in vivo. However, the means by which meiotic resumption is regulated remains unclear. cAMP within the oocyte has been shown to play a critical role in maintaining oocyte meiotic arrest. High levels of cAMP prevent oocyte spontaneous maturation in vitro, while a decrease in oocyte cAMP is associated with the resumption of meiosis (Schultz et al. 1983, Dekel et al. 1984). Currently, there are two models for how inhibitory levels of oocyte cAMP are maintained. The first is that cAMP diffuses from cumulus cells to the oocyte through the gap junctions that couple the two cell types (Dekel et al. 1981). It follows that LH stimulation disrupts the interaction between oocyte and the follicle cells (Dekel 1988), leading to a decrease in oocyte cAMP and subsequent re-entry into the cell cycle (Edry et al. 2006). Alternatively, studies have shown that the oocyte can generate cAMP by the constitutive activity of the heterotrimeric G protein $\mathrm{G}_{\mathrm{s}}$ (Mehlmann et al. 2002, Kalinowski et al. 2004, Freudzon et al. 2005) and, subsequently, type 3 adenyl cyclase (AC3; Horner et al. 2003). Inhibition of $G_{s}$ by injection of follicle-enclosed oocytes with a $\mathrm{G}_{\mathrm{s}}$ inhibitory antibody or a dominantnegative form causes meiotic resumption in the intact follicle (Mehlmann et al. 2002). A later study showed that it is GPR3, an orphan member of the G proteincoupled receptor family, that constitutively activates $\mathrm{G}_{\mathrm{s}}$ protein and maintains oocyte cAMP levels (Mehlmann et al. 2004). In rat oocytes, AC3 is the major cyclase isoform that produces CAMP, and mouse oocytes deficient in AC3 show defective meiotic arrest in vivo and accelerated spontaneous maturation in vitro (Horner et al. 2003), indicating the involvement of AC3 in the control of oocyte meiotic arrest. Therefore, based on these observations, to induce oocyte maturation, a positive signal should either reduce oocyte cAMP or overcome its inhibition.

FSH stimulates the maturation of cumulus cellenclosed oocytes (CEOs) in vitro (Downs et al. 1988). 
FSH also produces a transient arrest of spontaneous oocyte maturation. Following FSH stimulation, the cAMP concentration transiently rises in oocyte-cumulus cell complexes (Schultz et al. 1983, Salustri et al. 1985) and a parallel increase is observed in oocytes (Salustri et al. 1985, Webb et al. 2002), suggesting that the increase in oocyte cAMP is responsible for the delay in meiotic resumption. It must be noted that FSH-treated CEOs resume meiosis before oocyte cAMP decreases to basal levels (Salustri et al. 1985), suggesting the presence of an $\mathrm{FSH}$-induced meiotic signal that overcomes the cAMP-regulated meiotic arrest.

Cyclic nucleotide phosphodiesterase (PDE) is the enzyme that degrades CAMP and is essential for driving oocyte meiotic resumption by lowering inhibitory cAMP levels. In rodents, oocyte cAMP hydrolysis is primarily carried out by PDE3A (Richard et al. 2001, Shitsukawa et al. 2001). PDE3A activity increases in rodent oocytes before meiotic resumption in both spontaneous and gonadotropin-stimulated maturation (Richard et al. 2001), and inhibition of the oocyte PDE3 activity completely blocks oocyte maturation in vitro and in vivo (Wiersma et al. 1998, Conti et al. 2002). PDE3A null oocytes lack cAMP-specific PDE activity, contain increased cAMP levels, and fail to undergo spontaneous maturation in vitro (Masciarelli et al. 2004). Recently, in mouse oocytes, protein kinase B/AKT has been shown to regulate PDE3A activity by phosphorylation on the Ser290-292 sites, thus playing a role in the regulation of oocyte meiotic induction (Han et al. 2006).

AMP, a product of ATP and cAMP degradation, is an important factor regulating AMP-activated protein kinase (PRKA) activity. PRKA is a cellular energy sensor composed of a catalytic $\alpha$ subunit, and regulatory $\beta$ and $\gamma$ subunits (Hardie \& Hawley 2001). AMP allosterically binds to the $\beta$ subunit and facilitates the activation of the enzyme (Davies et al. 1995, Hawley et al. 2002, Sanders et al. 2007). It has been shown that PRKA activation in mouse oocytes provides a positive stimulus for meiotic resumption in vitro in response to a variety of nonphysiological and physiological signals (Chen et al. 2006, Chen \& Downs 2008). Here, it is proposed that a transient increase of cAMP concentration in oocytes could provide a source of AMP, which activates PRKA, inducing oocytes to overcome meiotic inhibition and re-enter meiosis.

To test this hypothesis, denuded oocytes (DO) were pulsed with CAMP and then assessed for meiotic maturation and PRKA activation. Our results indicate that an increase in CAMP within the oocyte can induce meiotic resumption if the culture conditions allow subsequent CAMP degradation to AMP and activation of PRKA.

\section{Results}

\section{Pulsing oocytes with endogenous cAMP induces oocyte maturation and AMPK activation}

Apart from one experiment where pulsing time was varied, the experimental paradigm for this study was to pretreat, or pulse, DO for $3 \mathrm{~h}$ with either cAMP analogs or cAMP-elevating agents, wash out the pulsing agent, and then continue culture in meiotic-arresting medium before assessing the parameter in question. Initial experiments utilized the cAMP-elevating agent, forskolin, to test the effects of endogenous cAMP. Forskolin activates $A C$ by directly interacting with the catalytic subunit of the enzyme, and has been shown to raise cAMP levels in mouse oocytes (Schultz et al. 1983, Urner et al. 1983). DO were maintained in meiotic arrest with $1 \mathrm{mM} \mathrm{N} \mathrm{N}^{6}$-monobutyryladenosine cAMP (mbcAMP), and exposed to increasing concentrations of forskolin. After washing out the forskolin, culture in mbcAMP was continued for 17-18 h before maturation assessment. Oocytes continuously cultured in mbcAMPcontaining medium without forskolin for 20-21 h served as controls. Oocyte maturation was dosedependently induced by forskolin pulsing, compared to oocytes cultured in mbcAMP alone (an increase from 17 to $34 \%$ germinal vesicle breakdown (GVB), Fig. 1A), while continuous exposure to forskolin had no stimulatory effect.

We next tested the time course of meiotic maturation after forskolin pulsing under these conditions. DO were cultured $3 \mathrm{~h}$ in $1 \mathrm{ml}$ medium in culture tubes containing mbcAMP alone or mbcAMP plus $100 \mu \mathrm{M}$ forskolin. The mbcAMP-treated oocytes were then transferred to the same medium, while oocytes pulsed in mbcAMP plus forskolin were split into two groups: one was transferred to the same medium, while the other was washed free of forskolin and transferred to mbcAMP-containing medium. All three secondary cultures were carried out in $200 \mu \mathrm{l}$ medium under oil so periodic maturation assessment could be made after 2, 5, 8 and $18 \mathrm{~h}$ of culture. When cultured in mbAMP alone, maturation gradually increased from 11 to $43 \%$ GVB (Fig. 1B). Continuous exposure to forskolin completely suppressed GVB. Oocytes pulsed with forskolin had a small increase $(10 \%)$ in maturation between 0 and $2 \mathrm{~h}$, but a $30 \%$ increase was manifest between 2 and $5 \mathrm{~h}$, with more modest increases resuming thereafter. The frequency of maturation in pulsed oocytes surpassed that of the mbcAMP controls during the $2-5 \mathrm{~h}$ post-pulse period, but the difference was never $>12 \%$.

A general PDE inhibitor, 3-isobutyl-1-methylxanthine (IBMX), has been used to promote CAMP accumulation by preventing its degradation. Oocytes treated with both forskolin and IBMX would be expected to contain higher levels of cAMP than forskolin alone; consequently, pulsing oocytes with the two compounds together might be more effective in meiotic induction than 

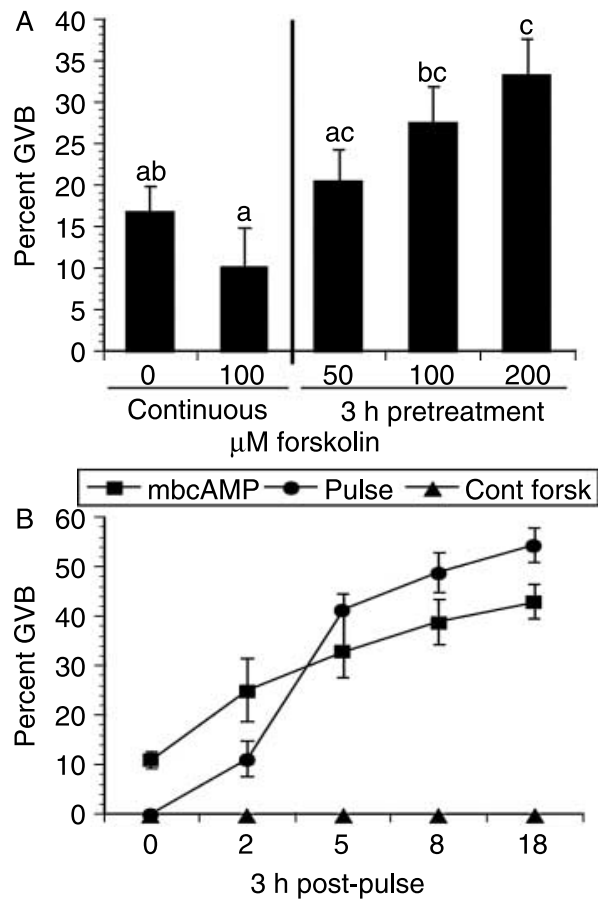

Figure 1 Effects of forskolin-pulsing on oocyte meiotic resumption. (A) Forskolin dose-response. Denuded oocytes (DO), maintained in meiotic arrest with $1 \mathrm{mM}$ mbcAMP, were pulsed with increasing concentrations of forskolin for $3 \mathrm{~h}$, washed free of forskolin, and cultures were continued in mbcAMP alone for 17-18 $\mathrm{h}$ before assessment of GVB. Other oocytes were continuously cultured with or without $100 \mu \mathrm{M}$ forskolin in the meiotic-arresting medium for 20-21 h. Groups with no common letter are significantly different.

(B) Maturation kinetics. Oocytes were treated as in (A) for $3 \mathrm{~h}$; then, post-pulse cultures were carried out in medium drops under oil with meiotic maturation assessed after the designated time periods.

pulsing with either agent alone. Thus, mbcAMP-arrested DO were pulsed with $100 \mu \mathrm{M}$ forskolin plus $100 \mu \mathrm{M}$ IBMX, washed, and returned to medium containing only mbcAMP for 17-18 h. Under these conditions, maturation was again stimulated, but to no greater extent than pulsing with forskolin alone (an increase of 17\% from 37 to $54 \%$ GVB; Fig. 2A). Continuous exposure to forskolin plus IBMX reduced the maturation by $29 \%$.

Active AMPK (PRKA) specifically phosphorylates Ser79 of acetyl-CoA carboxylase (ACACA); thus, western analysis using anti-phospho-ACACA antibody was performed to indirectly detect PRKA activity in the pulsed oocytes. Cellular extracts were obtained from GV-stage oocytes after pulsing with IBMX plus forskolin and $2 \mathrm{~h}$ additional culture in mbcAMP-containing medium, or after $5 \mathrm{~h}$ continuous treatment in mbcAMP alone (control) or mbcAMP plus IBMX and forskolin, and were then processed for western analysis. The intensity of pACACA bands was quantified and normalized to ACACA levels. pACACA band intensity was increased by $33 \%$ in oocytes after pulsing with IBMX plus forskolin compared to oocytes continuously cultured in mbcAMPcontaining medium (Fig. 2B), indicating that PRKA was activated in the pulsed oocytes before meiotic resumption. Continuous exposure to forskolin plus IBMX had no effect on band intensity.

To determine if the meiosis-inducing action of forskolin would occur under different inhibitory conditions, the dose-response experiment was repeated in $4 \mathrm{mM}$ hypoxanthine (HX)-supplemented medium. Although meiotic arrest was maintained to a lesser extent (58\% GVB), maturation was again stimulated by forskolin pulsing, resulting in a $26 \%$ increase to $84 \%$ GVB; Fig. 2A). On the other hand, continuous exposure to $\mathrm{HX}$ plus forskolin resulted in a significant reduction in maturation (14\% GVB).

The time course of meiotic induction by forskolin pulsing in HX-arrested oocytes was next tested by
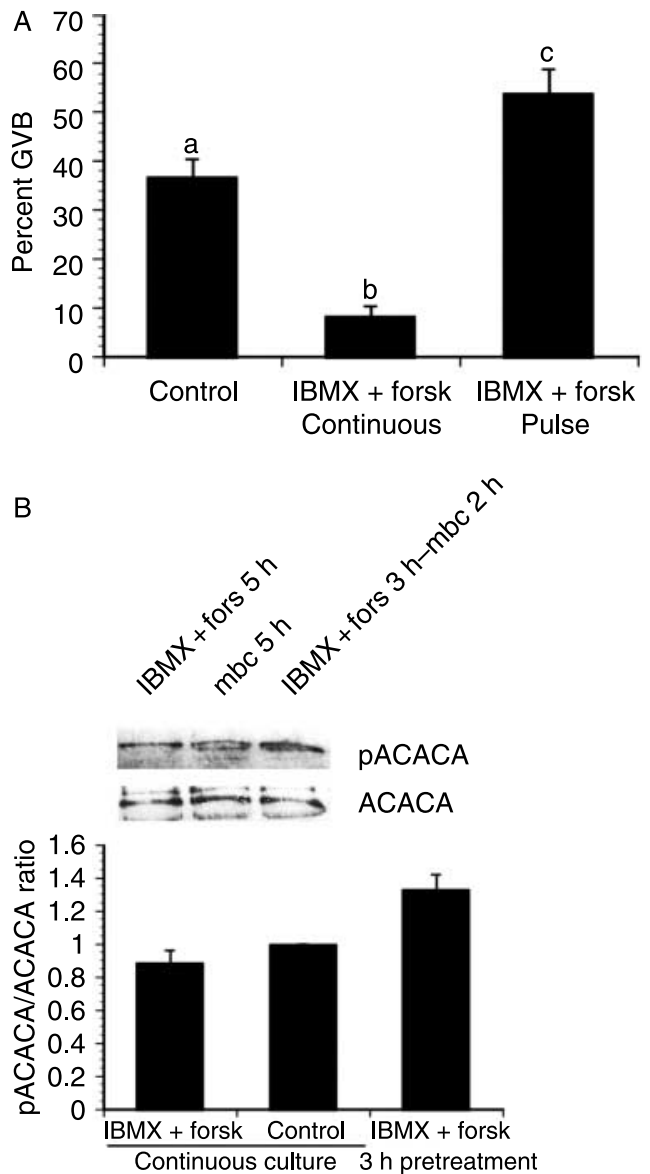

Figure 2 Effects of pulsing oocytes with forskolin and IBMX on oocyte maturation and PRKA activation. (A) DO were cultured for 21-22 $\mathrm{h}$ in $1 \mathrm{mM}$ mbcAMP or mbcAMP plus forskolin $(100 \mu \mathrm{M})$ and IBMX $(100 \mu \mathrm{M})$; a third group was pulsed $3 \mathrm{~h}$ with forskolin plus IBMX, washed free of these agents and then cultured a further $17-18 \mathrm{~h}$ in mbcAMP alone before assessing meiotic status. Groups with no common letter are significantly different. (B) DO were exposed to the same treatments as in (A) except that GV-stage oocytes were collected after $5 \mathrm{~h}$ and the extracts were processed for western analysis (300 oocytes per lane), using antibody to phospho-ACACA. The mean PACACA/ACACA ratio of four blots is shown, normalized to the mbcAMP alone group. 
means identical to those used earlier with mbcAMParrested oocytes. When cultured in HX alone, a low level of maturation was maintained throughout the secondary culture period (an increase from $9 \%$ at $0 \mathrm{~h}$ to $30 \%$ GVB after $18 \mathrm{~h}$ ). Inhibition in oocytes exposed continuously to HX plus forskolin was more pronounced, with a small increase only after $8 \mathrm{~h}$ (from 0 to $13 \%$ GVB; Fig. 3B). Oocytes pulsed with HX plus forskolin and washed free of the forskolin exhibited an increase in maturation compared to oocytes cultured only in HX, and this was first manifested between 2 and $5 \mathrm{~h}$ of culture post-pulse with a $26 \%$ increase by $18 \mathrm{~h}$ (Fig. 3B).

To confirm that forskolin-pulsed oocytes produced a transient increase in CAMP, cyclic nucleotide concentrations were measured by direct cAMP enzyme immunoassay. After pulsing with $100 \mu \mathrm{M}$ forskolin in $4 \mathrm{mM} \mathrm{HX}$, oocytes were collected or washed free of forskolin and cultured in HX-containing medium for an additional 2 or $5 \mathrm{~h}$ before assay. A parallel group of oocytes was continuously cultured in the HX-containing medium for the same treatment time, and an additional group was maintained in the presence of $\mathrm{HX}$ plus forskolin for $8 \mathrm{~h}$. HX, a weak PDE inhibitor (Downs et al. 1989), maintained intra-oocyte cAMP concentration at similar levels (0.17-0.19 fmol/oocyte) during the culture (Fig. 3C). After forskolin treatment for $3 \mathrm{~h}$, oocyte cAMP concentration was elevated from 0.24 to $0.33 \mathrm{fmol} /$ oocyte and the concentration was even higher after $8 \mathrm{~h}$ of forskolin treatment $(0.43 \mathrm{fmol} /$ oocyte). When oocytes were cultured for $2 \mathrm{~h}$ in the control medium after forskolin pulsing, the cAMP concentration was dramatically decreased to $0.11 \mathrm{fmol} /$ oocyte, with no further decrease after 3 additional hours of culture in HX-containing medium.

To examine the effects of forskolin pulsing on the generation of AMP, individual oocytes were collected and assayed for AMP. Freshly isolated oocytes contained $1.70 \mathrm{mmol}$ AMP per kg wet weight of oocyte (Fig. 3D). After $3 \mathrm{~h}$ culture in medium containing HX or HX plus
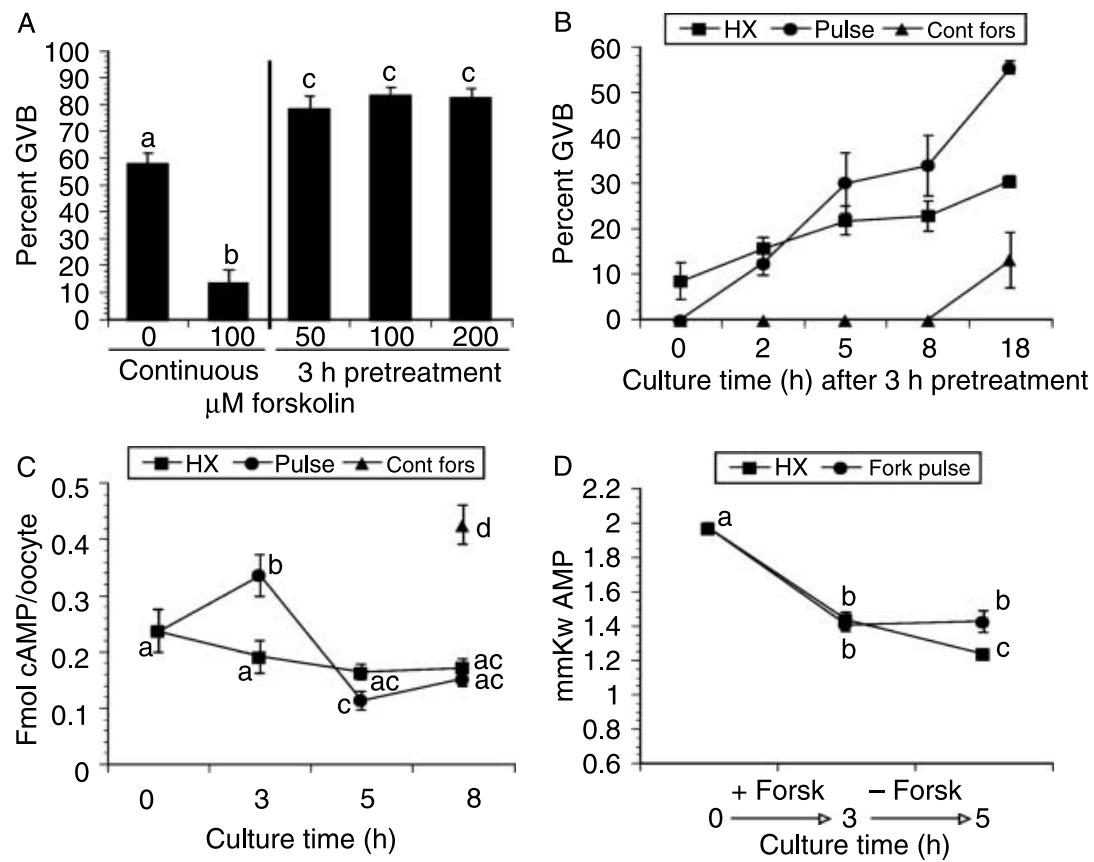

Figure 3 Effects of forskolin pulsing on oocyte maturation and cAMP and AMP concentrations in hypoxanthine (HX)-arrested oocytes. (A) Forskolin dose-response. Hypoxanthine-arrested DO were pulsed $3 \mathrm{~h}$ with increasing concentrations of forskolin, washed free of forskolin and returned to $\mathrm{HX}$-containing medium; alternatively, they were cultured continuously in HX alone or HX plus $100 \mu \mathrm{M}$ forskolin. GVB was assessed after a total of 21-22 h. (B) Maturation kinetics. After $3 \mathrm{~h}$ pretreatment with HX plus $100 \mu \mathrm{M}$ forskolin, DO were washed and cultured in HX alone. Meiotic resumption was determined at the indicated culture times after pulsing. Maturation kinetics was also determined for oocytes continuously cultured in HX alone or HX plus $100 \mu \mathrm{M}$ forskolin (Fors). Time $0 \mathrm{~h}$ represents $3 \mathrm{~h}$ of culture. (C) cAMP assay. Fresh DO were collected (time 0 ) or DO were cultured in $4 \mathrm{mM} \mathrm{HX}$-containing medium in the presence (Fors pulse) or absence (HX) of $100 \mu \mathrm{M}$ forskolin. Oocytes in the HX group were collected after 3,5 , and $8 \mathrm{~h}$ of culture. Oocytes in the Fors pulse group were washed free of forskolin after $3 \mathrm{~h}$ and returned to HX-containing medium. Oocytes from this group were collected immediately after the forskolin pulse or 2 or $5 \mathrm{~h}$ after washing out the forskolin and subsequent culture in $\mathrm{HX}(3,5$, and 8 total hours of culture respectively). An additional group of oocytes was continuously cultured in HX plus forskolin for the entire 8-h period (Cont Fors). Only GV-stage oocytes were assayed for cAMP. Results shown here represent the mean \pm s.E.M. of seven experiments. Groups with no common letter are significantly different. (D) AMP assay. Fresh oocytes were collected or DO were cultured in medium containing HX in the presence or absence of forskolin. Oocytes treated with HX alone were collected after 3 and $5 \mathrm{~h}$ of culture. Oocytes treated with HX plus forskolin were collected $3 \mathrm{~h}$ after forskolin pulsing and $2 \mathrm{~h}$ after pulsed oocytes were washed free of forskolin and returned to HX medium $(5 \mathrm{~h}$ total culture time). Groups with no common letter are significantly different. 
forskolin, oocyte AMP concentration was decreased to 1.44 or $1.41 \mathrm{mmol} / \mathrm{kg}$ respectively. Oocytes cultured in $\mathrm{HX}$ for $5 \mathrm{~h}$ exhibited a further decline in AMP concentration to $1.24 \mathrm{mmol} / \mathrm{kg}$; however, the 3 -h pulse with forskolin prevented this further decline with AMP maintained at $1.43 \mathrm{mmol} / \mathrm{kg}$. The results are consistent with production of AMP as CAMP levels decline following forskolin pulsing.

\section{Pulsing oocytes with 8-bromo-cAMP induces oocyte maturation and $A M P K$ activation}

8-bromo-cAMP (8-Br-cAMP), a PDE sensitive cAMP analog, can be metabolized to 8-Br-AMP. Oocytes were pulsed with increasing concentrations of 8-Br-cAMP in mbcAMP-containing medium, washed, and cultured in mbcAMP alone for an additional 17-18 h before assessing GVB. Oocytes continuously cultured in the meiosis-arresting medium without 8-Br-cAMP for $20-21 \mathrm{~h}$ served as controls. As shown in Fig. 4A, the maturation percentage was induced in $6 \mathrm{mM}$ 8-Br-cAMP-pulsed oocytes (a 22\% increase), compared to the control groups. Continuous culture in 8-Br-cAMP had no effect on the maturation percentage.

To establish how pulsing time affects meiotic induction, mbcAMP-arrested oocytes were pretreated with $6 \mathrm{mM} 8$-Br-cAMP for 1, 3, or $5 \mathrm{~h}$ before subsequent culture in mbcAMP-arresting medium. The total culture time was $20-21 \mathrm{~h}$. Figure $4 \mathrm{~B}$ shows that a pulse time of 3 or $5 \mathrm{~h}$, but not $1 \mathrm{~h}$, significantly induced meiotic maturation compared to oocytes not exposed to 8-Br-cAMP (an increase of 26\%).

When HX-arrested oocytes were similarly pulsed with $6 \mathrm{mM} 8$-Br-cAMP, maturation was stimulated by $18.5 \%$ (Fig. 4C). Continuous exposure to 8-Br-cAMP was again without effect.

To examine PRKA activation in pulsed oocytes, mbcAMP-arrested DO were exposed to 8-Br-cAMP, washed, and then returned to mbcAMP-containing medium for $2 \mathrm{~h}$. These oocytes were fixed and stained with antibody to phospho-PRKA for detection by immunofluorescence. Oocytes cultured $5 \mathrm{~h}$ in mbcAMP alone or mbcAMP plus 8-Br-cAMP were also processed in this manner. Figure $5 \mathrm{~A}$ shows the GV area from two oocytes from the pulsed group, each stained with DAPI (blue) for DNA and with FITC (green) for active PRKA. In the oocyte to the left, PRKA has not been stimulated; however, punctate staining for PRKA is apparent in the oocyte to the right, indicating active kinase.

The frequency of nuclear staining for the three groups was next quantified, with these data presented in Fig. 5B. A mean of $34 \%$ of oocytes cultured $5 \mathrm{~h}$ in mbcAMP contained nuclei that stained positively for active PRKA, while the addition of 8-Br-cAMP had no significant effect $(22 \%)$. However, pulsing with 8 -Br-cAMP produced a significant increase in oocytes with positive nuclear staining $(62 \%)$.
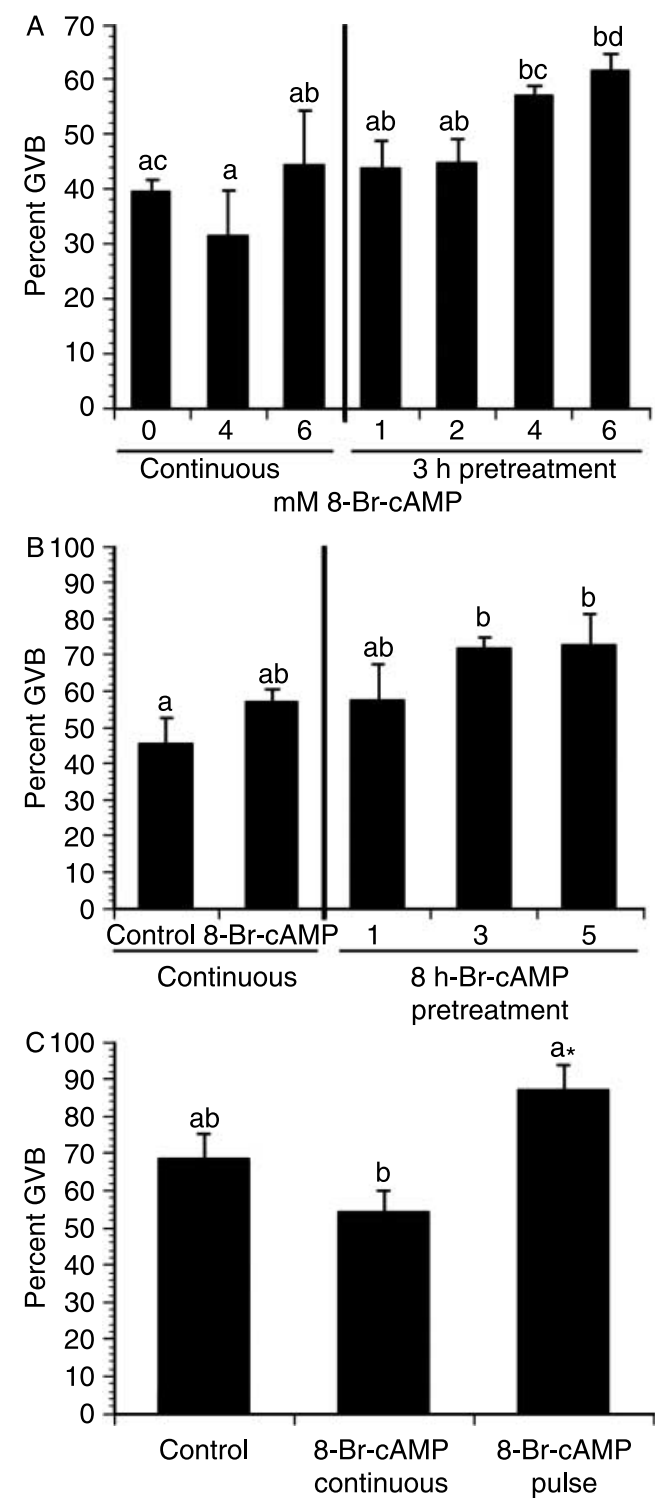

Figure 4 Effects of 8-Br-cAMP pulsing on oocyte maturation. (A) Doseresponse effect of 8 -Br-cAMP treatment. DO were pretreated for $3 \mathrm{~h}$ in $1 \mathrm{mM}$ mbcAMP with increasing concentrations of 8-Br-cAMP before they were washed free of 8-Br-cAMP and cultured 17-18 $\mathrm{h}$ in mbcAMP alone. Separate sets of oocytes were cultured for 21-22 h in either mbcAMP alone or mbcAMP plus 4 or $6 \mathrm{mM} 8$-Br-cAMP. Groups with no common letter are significantly different. (B) Time-dependent effect of 8-Br-cAMP pretreatment. DO were pulsed with $6 \mathrm{mM}$ 8-Br-cAMP for the indicated times before culture in $1 \mathrm{mM}$ mbcAMP. The total culture time (including the pulsing time) was 20-21 h. Oocytes continuously cultured in mbcAMP or mbcAMP plus $6 \mathrm{mM}$ 8-Br-cAMP for 20-21 h served as controls. Groups with no common letter are significantly different. (C) Effect of 8-Br-cAMP pulsing on HX-arrested oocytes. DO cultured in $4 \mathrm{mM} \mathrm{HX}$ were pulsed $3 \mathrm{~h}$ with $6 \mathrm{mM}$ 8-Br-cAMP, washed free of the cyclic nucleotide and cultured 17-18 additional hours in HX alone. Other oocytes were continuously cultured 21-22 h in HX alone or HX plus 8-Br-cAMP. Note that although the pulse group was not significantly different from the HX continuous group by ANOVA, it was by Student's $t$-test, as indicated by the asterisk. 
Western analysis was performed to detect pPRKA or pACACA in 8-Br-cAMP-pulsed GV-stage oocytes. GV-stage oocytes were pulsed with mbcAMP plus $6 \mathrm{mM}$ 8-Br-cAMP, washed, and cultured in mbcAMP alone for $2 \mathrm{~h}$ before they were collected for analysis. GV-stage oocytes were also collected after $5 \mathrm{~h}$ culture in mbcAMP-containing medium with or without 8-Br-cAMP. The blots were probed with either antipPRKA or anti-pACACA antibody. The intensity of the pPRKA band in 8-Br-cAMP pulsed oocytes was dramatically enhanced compared to the other two groups (Fig. 5C), indicating PRKA activation in the pulsed oocytes before meiotic maturation, and this is further indicated by quantitation of band intensity. Accordingly, the pACACA levels in the pulsed oocytes were also increased compared to the oocytes cultured in mbcAMPcontaining medium. However, surprisingly, oocytes
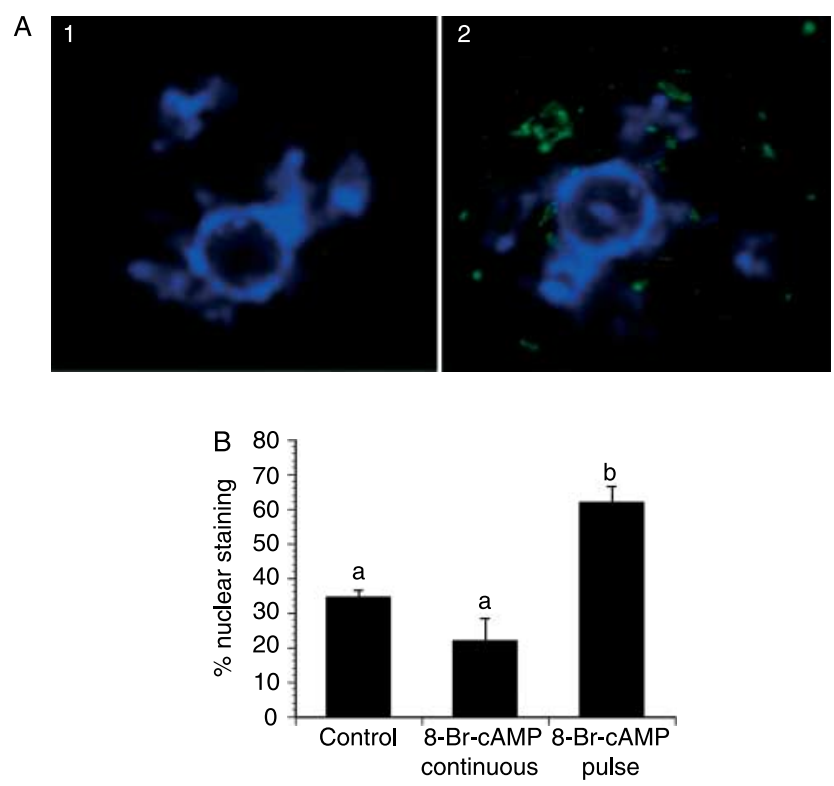

C
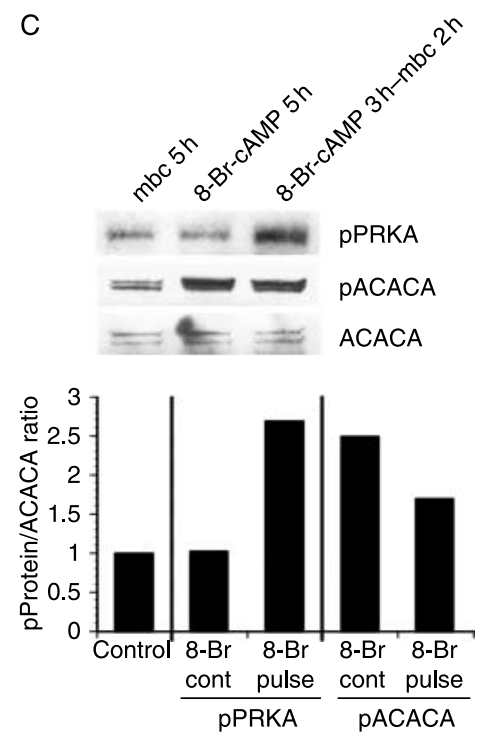

continuously cultured with 8-Br-cAMP contained high levels of pACACA but relatively low levels of pPRKA. The inconsistency of the two phosphorylated protein levels suggests that other mechanisms, besides active PRKA, may act to regulate ACACA phosphorylation on the Ser79 site.

To test whether PRKA activity is required for pulsinginduced maturation, pulsed oocytes were exposed to PRKA inhibitors. Compound C, a small molecular weight PRKA inhibitor (Zhou et al. 2001), and araA, a precursor of araATP (a competitive inhibitor of PRKA (Henin et al. 1996)), have been shown to block meiotic maturation via inhibition of PRKA activation (Chen et al. 2006). After pretreatment with $6 \mathrm{mM}$ 8-Br-cAMP, oocytes were washed and continuously cultured in the presence or absence of compound $\mathrm{C}$ or araA in mbcAMP-arresting medium for an additional 17-18 h. The two PRKA inhibitors, compound $\mathrm{C}$ and araA, both eliminated 8-Br-cAMP pulsing-induced maturation (Fig. 6A and B), thereby implicating PRKA activation in the CAMPpulsing-induced meiotic resumption.

\section{Pulsing with PDE-resistant cAMP analogs does not induce meiotic resumption}

To rule out the possibility that transiently increasing cAMP activates cAMP-dependent protein kinase (PRKACA) that triggers meiotic resumption by means unrelated to PRKA activation, oocytes were pulsed with PDE-resistant CAMP analogs in $1 \mathrm{mM}$ mbcAMPcontaining medium. The cAMP analogs, cAMP acetoxymethyl ester, Sp-isomer (Sp-cAMP-AM) and 5,6dichloro-1-beta-D-ribofuranosylbenzimidazole-cAMP, Sp-isomer (Sp-5,6-DCl-cBIMPS), are activators of PRKACA, and both are resistant to PDE degradation

Figure 5 Effect of 8-Br-cAMP pretreatment on PRKA activation. (A) Fluorescent nuclear staining for phospho-PRKA. mbcAMP-arrested DO were pretreated with $6 \mathrm{mM}$ 8-Br-cAMP, washed free of 8-Br-cAMP, and then cultured $2 \mathrm{~h}$ in mbcAMPalone. GV-stage oocytes were processed for immunofluorescent staining using anti-phospho-PRKA antibody and FITC-labeled secondary antibody (green). DNA was stained with DAPI (blue). Panel 1 shows an oocyte with unstimulated PRKA, while the oocyte in panel 2 has active PRKA, indicated by punctate staining within the GV. Oocytes not exposed to primary antibody had only a faint diffuse staining throughout the oocyte and never displayed nuclear staining (not shown). (B) Quantification of germinal vesicle staining for phospho-PRKA. The frequency of nuclear (germinal vesicle) staining was compared in oocytes continuously cultured in mbcAMP or mbcAMP plus 8-br-cAMP with oocytes pulsed $3 \mathrm{~h}$ in mbcAMP plus 8-Br-cAMP followed by $2 \mathrm{~h}$ in mbcAMP alone. Groups with no common letter are significantly different. (C) Western analysis of 8-Br-cAMP-pulsed oocytes. DO treated identically to those in (B) above were processed for western analysis for pPRKA and pACACA (500 oocytes per lane). The mean pPRKA/ACACA and pACACA/ACACA ratios from two blots are shown normalized to the mbcAMP alone group. Note that pPRKA increases only after an 8-Br-cAMP pulse, while pACACA levels increase after either a pulse or continuous exposure to 8 -Br-cAMP. Shown are the mean values for two blots. 

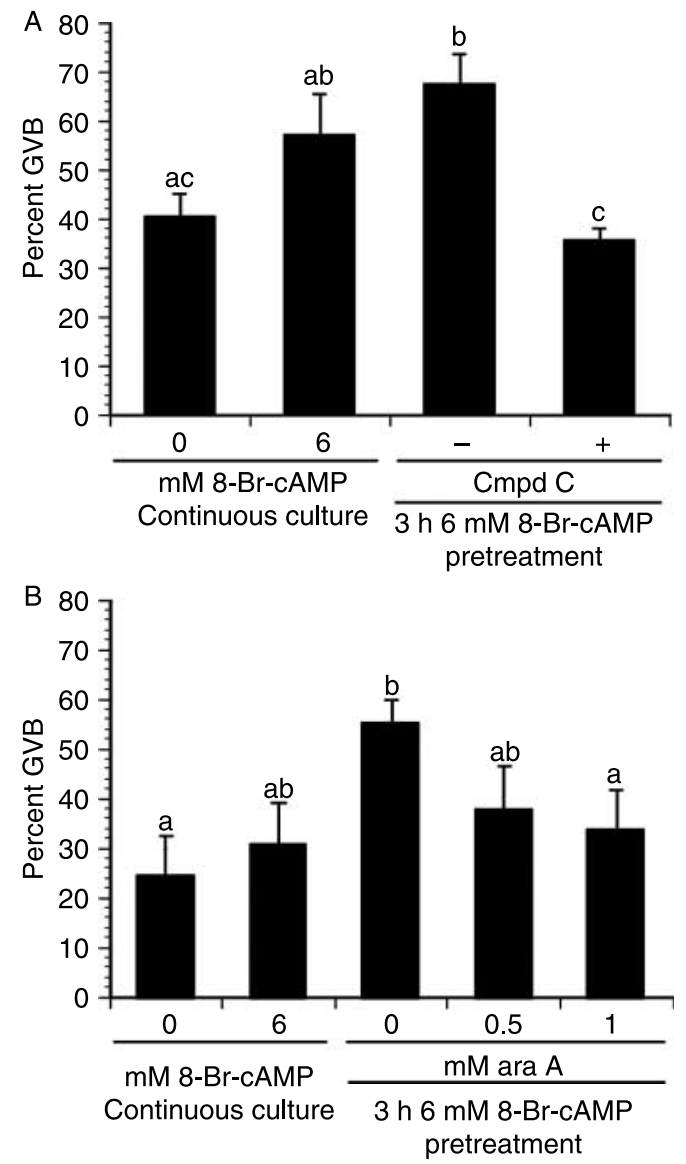

Figure 6 Effects of compound C (A) and araA (B) on 8-Br-cAMP pulsinginduced maturation. After $3 \mathrm{~h}$ pulsing with mbcAMP plus $6 \mathrm{mM}$ 8-Br-cAMP, DO were washed free of 8-Br-cAMP and cultured in mbcAMP alone or mbcAMP plus either compound $C(A)$ or araA $(B)$ for 17-18 h. Oocytes continuously cultured in mbcAMP or mbcAMP plus $6 \mathrm{mM} 8$-Br-cAMP served as controls. Groups with no common letter are significantly different.

(Dostmann et al. 1990, Sandberg et al. 1991). DO were pretreated with increasing concentrations of Sp-cAMPAM or Sp-5,6-DCl-cBIMPS in mbcAMP-arresting medium, washed free of the compound and continuously cultured in the control medium for $17-18 \mathrm{~h}$ before determining maturation percentage. Neither Sp-cAMPAM nor Sp-5,6-DCl-cBIMPS pretreatment induced oocyte maturation, compared to the continuously cultured groups (Fig. 7A and B). Both of these agents were used at effective doses, since continuous exposure to either augmented the mbcAMP-induced meiotic arrest by $>15 \%$.

Western analysis was carried out to detect pPRKA and pACACA in Sp-5,6-DCl-cBIMPS pulsed GV-stage oocytes. Little phosphorylation on the Thr172 site of PRKA or the Ser79 site of ACACA was observed after $3 \mathrm{~h}$ of Sp-5,6-DCl-cBIMPS pulsing plus $2 \mathrm{~h}$ culture in the control medium (Fig. 7C), indicating negligible activation of PRKA in oocytes after PDE-resistant cAMP analog pulsing.

\section{Pulsing oocytes with an exchange protein directly activated by CAMP-specific activator does not induce meiotic resumption}

cAMP signaling can be PRKACA-dependent or PRKACAindependent. Exchange protein directly activated by cAMP (Epac), another target of cAMP (de Rooij et al. 1998, Enserink et al. 2002), is a guanine nucleotide-exchange factor (GEF) for the Ras-like small GTPase Rapl and Rap2. To test whether transiently increasing CAMP levels within oocytes induces meiotic resumption through the activation of Epac, oocytes were pulsed with the Epac-specific activator 8-(4chlorophenylthio)-2-O-methyladenosine $3^{\prime}, 5^{\prime}$-cyclic monophosphate (8-CPT-2'-O-Me-cAMP; Enserink et al. 2002). Pulsing oocytes with increasing concentrations of 8-CPT-2'-O-Me-cAMP had no effect on oocyte meiotic maturation status compared to the continuously cultured controls (Fig. 8A). Yet 8-CPT-2'-O-Me-cAMP potentiated the meiosis-arresting effect of mbcAMP in the continuous cultures, indicating that oocytes are permeable to the compound. The results also suggest that the activation of Epac may contribute to oocyte meiotic arrest. To further examine this possibility, DO were cultured for $4 \mathrm{~h}$ in inhibitor-free medium with increasing concentrations of 8-CPT-2'-O-Me-CAMP. 8-CPT-2'-O-Me-cAMP at the concentration of $500 \mu \mathrm{M}$ did not show any inhibitory effect on oocyte spontaneous maturation (Fig. 8B), suggesting that Epac activation, though augmenting the effect of PRKACA, does not play an important role in cAMP-regulated oocyte meiotic arrest.

\section{Discussion}

Sustaining elevated levels of oocyte cAMP is important in maintaining meiotic arrest. Yet in this study we have shown that a transient increase of cAMP levels in DO can stimulate meiotic resumption through the activation of PRKA. Short-term elevation of intra-oocyte cAMP concentrations by forskolin induced oocyte maturation, and this was associated with active PRKA as determined by western analysis. Meiotic induction and AMPK activation were also stimulated by pulsing oocytes with 8-Br-cAMP, a PDE-sensitive cAMP analog, and the meiosis-inducing effect was blocked by PRKA inhibitors. On the other hand, pulsing oocytes with the PDE-resistant cAMP analogs, Sp-cAMP-AM or Sp-5,6DCl-cBIMPS, had no effect on oocyte maturation and PRKA activation. Nor did pulsing with the Epac-specific activator, 8-CPT-2'-O-Me-cAMP, positively influence maturation suggesting that the meiosis-inducing effect of cAMP pulsing does not involve Epac.

Intra-oocyte CAMP levels are regulated by the activity of AC and PDE. Forskolin, an AC activator, has been shown to increase cAMP levels in mouse oocytes (Schultz et al. 1983, Urner et al. 1983). Consistent with this, after $3 \mathrm{~h}$ of forskolin treatment, oocyte cAMP 
levels were increased from 0.24 to 0.34 fmol/oocyte. HX is a meiotic inhibitor present in the follicular fluid (Downs et al. 1985, Eppig et al. 1985) that maintains meiotic arrest by preventing cAMP degradation (Downs et al. 1989). Oocyte cAMP decreased by $20 \%$ after $3 \mathrm{~h}$ in $\mathrm{HX}$, and stabilized $2 \mathrm{~h}$ later after a further $10 \%$
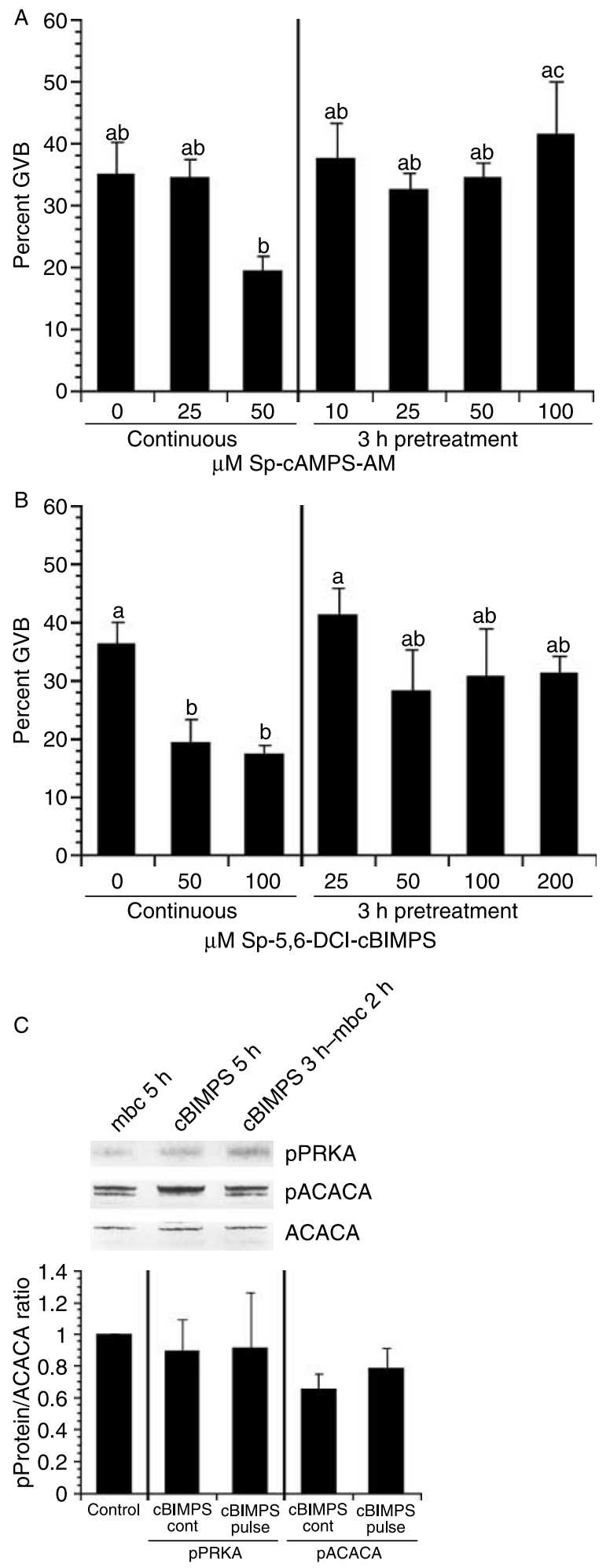

Reproduction (2009) 138 759-770 decline. This inability to completely prevent cAMP degradation renders $\mathrm{HX}$ a relatively weak meiotic inhibitor. When pulsed oocytes were washed free of forskolin and cultured in HX-containing medium for $2 \mathrm{~h}$, a dramatic drop in cAMP (from 0.34 to $0.11 \mathrm{fmol} /$ oocyte) was observed (Fig. 3A). Moreover, these oocytes contained a significantly higher level of AMP $(1.43 \mathrm{mmol} / \mathrm{kg}$ ) when compared to oocytes continuously cultured in HX alone $(1.24 \mathrm{mmol} / \mathrm{kg}$, Fig. 3B); thus, the decrease in cAMP can be accounted for, at least in part, by an increase in AMP. Moreover, these changes were occurring at the same time the maturation percentage in pulsed oocytes was beginning to rise above that in control oocytes. This is consistent with the activation of PRKA, as determined by western analysis, and accompanying stimulatory action on meiotic resumption. These data therefore demonstrate that cAMP pulsing can generate a stimulus to induce oocyte maturation and that PRKA is activated before GVB. However, it must be noted that oocyte cAMP levels within DO decreased below the initial concentration $2 \mathrm{~h}$ after forskolin pulsing, which may result in some inactivation of PRKACA and contribute to the meiotic resumption by a means unrelated to PRKA activation.

AMP activates PRKA by allosterically binding to the enzyme and preventing phosphatase-mediated dephosphorylation (Davies et al. 1995, Sanders et al. 2007). 8-Br-cAMP, a PDE-sensitive cAMP analog, can be degraded to 8-Br-AMP, which is a partial agonist at the allosteric binding site of AMP (Davies et al. 1989) and can activate PRKA at low concentrations. Pulsing oocytes with $6 \mathrm{mM}$ 8-Br-cAMP activated PRKA in GV-stage oocytes and induced meiotic maturation in mbcAMP-arrested, as well as HX-arrested, oocytes. Two PRKA inhibitors, compound $\mathrm{C}$ and araA, that prevent PRKA-induced meiotic maturation in vitro in mouse oocytes (Chen et al. 2006), blocked 8-Br-cAMP pulsinginduced maturation, further implicating PRKA activity in the meiotic response.

Acetyl CoA carboxylase (ACACA), as an important substrate of PRKA, becomes phosphorylated on Ser79 and thereby is inactivated ( $\mathrm{Ha}$ et al. 1994). Studies have shown that ACACA phosphorylation on Ser79

Figure 7 Effects of PDE-resistant cAMP analog pulsing on oocyte maturation. mbcAMP-arrested DO were pretreated $3 \mathrm{~h}$ with increasing concentrations of Sp-CAMP-AM (A) or SP-5,6-DCl-cBIMPS (B) and then washed and cultured in mbcAMP alone for 17-18 $\mathrm{h}$ before assessment of GVB. Oocytes continuously cultured in mbcAMP or mbcAMP plus Sp-cAMP-AM (A) or SP-5,6-DCl-cBIMPS (B) for 20-21 h served as controls. Groups with no common letter are significantly different. (C) Western analysis of pPRKA and pACACA in SP-5,6-DCl-cBIMPS-pulsed GV-stage oocytes. After $3 \mathrm{~h}$ pretreatment in $1 \mathrm{mM}$ mbcAMP plus $6 \mathrm{mM}$ SP-5,6-DCl-cBIMPS, DO were washed and cultured $2 \mathrm{~h}$ in mbcAMP. DO cultured in mbcAMP or mbcAMP plus $200 \mu \mathrm{M}$ SP-5,6-DCI-cBIMPS for $5 \mathrm{~h}$ served as controls. The mean pPRKA/ACACA and PACACA/ACACA ratios from four blots are shown normalized to the mbcAMP alone group. 


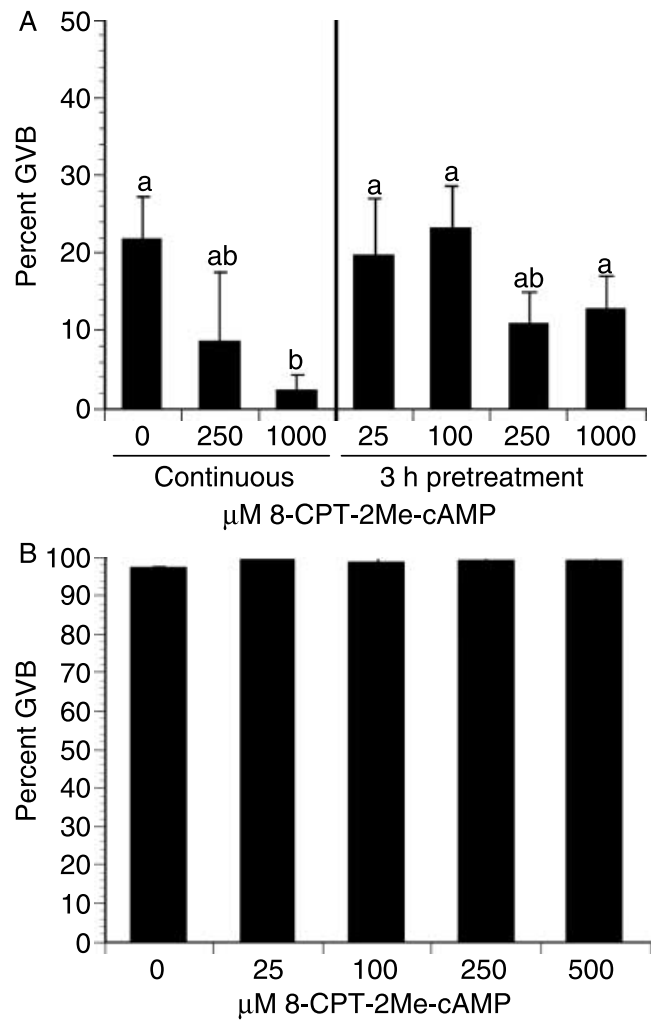

Figure 8 Effects of 8-CPT-2Me-cAMP on oocyte maturation. (A) DO were pulsed with increasing concentrations of 8-CPT-2Me-cAMP in $1 \mathrm{mM}$ mbcAMP for $3 \mathrm{~h}$. Oocytes were then washed and cultured in mbcAMP for 17-18 h before assessment of GVB. DO continuously cultured in mbcAMP or mbcAMP plus 8-CPT-2Me-cAMP served as controls. Groups with no identical letter are significantly different. (B) DO were cultured in inhibitor-free medium plus increasing concentrations of 8-CPT-2Me-cAMP. After $3 \mathrm{~h}$ of culture, the frequency of oocytes resuming maturation was determined.

corresponds to PRKA activation in rat muscle, liver, adipose tissue (Ruderman et al. 2003), and mouse oocytes (Chen et al. 2006). Therefore, ACACA phosphorylation has been widely used as an indicator of PRKA activity. However, in this study, oocytes continuously cultured with 8-Br-cAMP contained a high level of pACACA but relatively low levels of pPRKA, suggesting ACACA can be phosphorylated by means other than active PRKA. One possible candidate is active PRKACA. Haystead et al. (1990) reported that the activation of PRKACA by forskolin, IBMX or cAMP analogs ( $N^{6}$-butyryl cAMP plus 8-thiomethyl cAMP), leads to ACACA Ser79 phosphorylation and inactivation in isolated rat adipocytes. However, the mechanism of the observation is still not known, as PRKACA does not directly phosphorylate ACACA at the Ser 79 site (Ha et al. 1994). It is important to mention that the other CAMP analogs used in this study failed to increase pACACA levels in the pulsed oocytes, making it unlikely that this response is simply due to an increase in pPRKACA activity. In our experimental system, apart from this exception, pACACA levels were correlated with pPRKA in oocytes and directly related to the extent of meiotic induction. How persistent 8-Br-cAMP treatment results in ACACA phosphorylation remains unclear in this system. Nevertheless, it should be noted there was a trend towards increased meiotic resumption when oocytes were continuously cultured in medium containing high levels of $8-\mathrm{Br}-\mathrm{cAMP}$, raising the possibility that the phosphorylation state of ACACA influences meiotic regulation. Indeed, results of preliminary experiments suggest that suppression of ACACA activity, which would occur under PRKA activation, can lead to meiotic resumption in mouse oocytes.

Apart from PRKA activation, it was possible that cAMP pulsing-induced maturation is due to a transient hyperactivation of PRKACA that stimulates additional pathways capable of inducing meiotic resumption. To exclude this possibility, oocytes were pulsed with the PDE-resistant PRKACA activators, Sp-CAMP-AM and Sp-5,6-DCl-cBIMPS. Pulsing with these cAMP analogs had no effect on oocyte maturation or, in the case of Sp-5,6-DCL-cBIMPS, PRKA activation, suggesting that degradation of CAMP to AMP is required for pulsinginduced maturation. That these analogs were able to activate PRKACA is suggested by their inhibitory effect on maturation upon continuous exposure. Hence, PDE degradation of cAMP appears to be essential for pulsinginduced maturation.

Casting further doubt on a PRKACA-mediated mechanism in response to cAMP pulsing are the results of a recent study showing inhibition of PRKA by forskolin and IBMX (Hurley et al. 2006). In mouse oocytes, persistent activation of PRKACA by forskolin or cAMP analogs negatively regulates both PRKA and meiotic maturation. Hence, PRKA may be one of the substrates for PRKACA in mouse oocytes, and modulation of its phosphorylation state is a potential mechanism whereby PRKACA negatively regulates GVB.

It is well accepted that cAMP exerts its effect mainly through the activation of PRKACA. However, de Rooij et al. (1998) report that CAMP also directly regulates Epac. Epac proteins are GEFs for the small GTPases, Rapl, and Rap2, that have been implicated in various cellular processes such as integrin-mediated cell adhesion and cell-cell junction formation, the control of insulin secretion and neurotransmitter release (Bos 2006). CAMP has been shown to regulate oocyte maturation through the activation of PRKACA, but the possible contribution of Epac activation to such regulation is still unknown. Pulsing oocytes with an Epac-specific activator, 8-CPT-2'-O-Me-cAMP, had no effect on meiotic maturation. Moreover, 8-CPT-2'-O-Me-cAMP at $500 \mu \mathrm{M}$ failed to arrest oocyte spontaneous maturation after $4 \mathrm{~h}$ of culture, although the cAMP analog was taken up by the oocyte, as shown by its augmentation of mbcAMP-maintained arrest. These data indicate that Epac does not play a major role in either CAMP-mediated 
meiotic arrest or cAMP pulsing-induced meiotic resumption.

Pulsing oocytes with forskolin or cAMP analogs exposes them to brief periods of elevated CAMP, and the results of previous studies suggest that this may be a common component of meiotic resumption in mammals. In mice, treatment of CEO with FSH or exposure of follicles to forskolin caused a transient rise in oocyte cAMP, with meiotic resumption occurring before cyclic nucleotides fell below the pretreatment levels (Hashimoto et al. 1985, Salustri et al. 1985). Similar results were seen in follicle-enclosed rabbit and hamster oocytes exposed to hCG or forskolin (Hubbard 1985, 1986, Yoshimura et al. 1992a, 1992b). In ovine and porcine oocytes, cAMP was transiently elevated by the gonadotropin surge, and did not decrease below the fresh, nonstimulated levels in the early stages of maturation (Moor \& Heslop 1981). Whether these brief fluxes in oocyte cAMP were causally related to meiotic resumption was not examined in these reports.

If the oocyte cAMP concentration in freshly isolated oocytes is sufficient to maintain meiotic arrest, our data support the idea that meiosis can resume without cAMP falling significantly below this level. Nevertheless, a decrease in CAMP is necessary for meiotic resumption to occur, since mouse oocytes lacking PDE fail to undergo GVB in vivo or in vitro (Masciarelli et al. 2004). In addition, PDE activity in mouse oocytes was recently observed to increase before meiotic maturation in vivo (Han et al. 2006), and it is possible that increased oocyte CAMP could provide a signal for such increased PDE activity. In the present study, the dramatic drop in oocyte cAMP following forskolin pulsing in vitro is certainly consistent with this possibility. Therefore, increased AMP may be generated in gonadotropin-stimulated GV-stage oocytes that could activate PRKA and contribute to meiotic induction. PRKA becomes activated in mouse oocytes in vivo prior to GVB (Chen \& Downs 2008), but the mechanism is not yet known.

We have presented the unique finding that direct pulsing of arrested DO with forskolin or PDE-sensitive CAMP analogs can stimulate meiotic resumption in a PRKA-dependent manner. Nevertheless, while we have recently shown that PRKA plays an important role in hormone-induced maturation of mouse oocytes (Chen \& Downs 2008), several experimental results in the present study suggest that cAMP pulsing alone within oocytes may not be the principal means by which PRKA activation and meiotic resumption are controlled in mice: 1) high levels of cAMP were required to stimulate maturation, 2) a cAMP pulse time more than $1 \mathrm{~h}$ was required for meiotic induction, 3) the kinetics of maturation following CAMP pulsing was slower than that observed in vivo, and 4) the extent of meiotic induction following a cAMP pulse was limited, never exceeding $32 \%$. These findings are reminiscent of the response of mouse oocytes to different stresses, in which a lower level of PRKA activity resulted in a more subdued meiotic response when compared to the PRKA activator, AICAR (LaRosa \& Downs 2006). It therefore appears that the extent of PRKA activation made possible by cAMP pulsing alone is limited and likely cannot account completely for the meiotic induction resulting from more physiological stimuli. It should also be emphasized that our experiments were carried out with DO, an artifactual situation far removed physiologically from conditions in vivo. Nevertheless, these results show that the meiosis-inducing action of cAMP pulsing is not restricted to the somatic compartment and demonstrate a potential signaling function for CAMP within the oocyte beyond simply stimulation of PRKACA and maintenance of meiotic arrest. Though perhaps not absolutely required for meiotic resumption, CAMP pulsing may increase the efficiency by which meiotic resumption is achieved. Yet, the extent to which such a signaling function is manifested, in situ, remains to be determined.

\section{Materials and Methods \\ Oocyte isolation and culture conditions}

Animals were raised in the research colony of the principal investigator (S M D). All experiments were carried out with prior approval of the Marquette University Institutional Animal Care and Use Committee.

C57BL/6J $\times$ SJL/J Fl mice, 19-23 days old, were used for all experiments. Mice were primed with $5 \mathrm{IU}$ equine choronic gonadotropin and killed 2 days later by cervical dislocation. Ovaries were removed and placed in the culture medium, and CEOs were obtained by puncturing large antral follicles with sterile needles. DO were prepared by repeated pipetting with a Pasteur pipette or by passage through mouth-operated small bore pipettes. Tubes were gassed with a humidified mixture of $5 \% \mathrm{CO}_{2}, 5 \% \mathrm{O}_{2}$, and $90 \% \mathrm{~N}_{2}$ and placed in a water bath at $37{ }^{\circ} \mathrm{C}$ for the duration of culture. For maturation kinetics experiments, oocytes were placed in $200 \mu \mathrm{l}$ drops of medium under oil and cultured in a similarly gassed water-jacketed incubator.

The culture medium used was Eagle's minimum essential medium with Earle's salts (Gibco), supplemented with $0.23 \mathrm{mM}$ pyruvate, penicillin, streptomycin sulfate and $3 \mathrm{mg} / \mathrm{ml}$ crystallized lyophilized BSA (ICN ImmunoBiologicals, Lisle, IL, USA) and buffered with $26 \mathrm{mM}$ bicarbonate.

\section{Western analysis}

DO and oocyte-cumulus cell complexes were washed in PBS $(\mathrm{pH} 7.4) / P V P(3 \mathrm{mg} / \mathrm{ml})$ plus protease inhibitors (Protease Inhibitors Cocktail Tablets, $1 \mathrm{mM}$ Na orthovanadate, $2 \mu \mathrm{g} / \mathrm{ml}$ pepstatin, $50 \mathrm{mM} \beta$-glycerophospate) and then added to an equal volume of $2 \times$ Laemmli's buffer containing $20 \%$ $\beta$-mercaptoethanol (BME). After heating at $95^{\circ} \mathrm{C}$ for $5 \mathrm{~min}$, samples were stored frozen at $-80^{\circ} \mathrm{C}$ until used for western blotting. For western analysis, proteins were electrophoresed on a $3-8 \%$ Tris-acetate mini gel (Invitrogen) for $1 \mathrm{~h}$ at $150 \mathrm{~V}$ 
and then transferred to nitrocellulose at $100 \mathrm{~V}$ for $1 \mathrm{~h}$. To obtain the sharpest bands for active PRKA (pPRKA) blots, samples were electrophoresed on a $4-12 \%$ bis-Tris SDS mini gel (Invitrogen) for $50 \mathrm{~min}$ at $100 \mathrm{~V}$ and then in a semi-dry system transferred to nitrocellulose at $200 \mathrm{~mA}$ for $2.5 \mathrm{~h}$ at $4{ }^{\circ} \mathrm{C}$. Blots were blocked with $5 \%$ nonfat milk for $2 \mathrm{~h}$ at room temperature and then incubated with primary antibodies (anti-pPRKA or anti-pACACA, 1:250) overnight at $4{ }^{\circ} \mathrm{C}$, washed three times in Tris-buffered saline (TBS pH 7.4) and incubated with HRP-conjugated IgG (1:2000, in 5\% nonfat milk) for $1 \mathrm{~h}$ at room temperature. After washing in TBS, detection was performed with Supersignal Western Dura Chemiluminescent Substrate (Pierce, Rockford, IL, USA). Blots were stripped ( $7 \mu \mathrm{l} / \mathrm{ml} \mathrm{BME,} \%$ SDS, room temperature $30 \mathrm{~min}$ ) and reprobed with ACACA antiserum (1:2000) as a loading control. Bands were quantified by UVP Biolmaging Systems (UVP, Inc., Upland, CA, USA).

Anti-pACACA antiserum was purchased from Upstate Biotechnology Inc. (Lake Placid, NY, USA); anti-ACACA antibodies were a kind gift from Grahame Hardie; antipPRKA antiserum was obtained from Cell Signaling Technology (Beverly, MA, USA).

\section{AMP/ATP level measurement}

DO were cultured for varying periods in organ culture dishes containing $1 \mathrm{ml}$ culture medium. After the designated culture times, individual oocytes were assayed for AMP as previously described (Ratchford et al. 2007).

\section{cAMP assay}

The level of cAMP in DO was determined using a Direct Cyclic AMP Enzyme Immunoassay Kit (Assay Designs, Ann Arbor, MI, USA). Samples were washed in $3 \mathrm{mg} / \mathrm{ml}$ MEM/BSA containing 0.2 mM IBMX immediately after culture. DO (about 150 per group) in a volume of $10 \mu \mathrm{l}$ were transferred to $90 \mu \mathrm{l}$ of $0.1 \mathrm{M}$ $\mathrm{HCl}$, and stored in $-80{ }^{\circ} \mathrm{C}$ until assay. During the assay, all samples were acetylated according to the procedure of the kit. Plates were read by an EL800 Universal Microplate Reader (BIO-TEK; Winooski, VT, USA), and data was reported by the software KCjunior (BIO-TEK). The cAMP concentrations were determined by AssayZap V3 (Biosoft, Cambridge, UK).

\section{Immunofluorescence}

Oocytes were fixed with $4 \%$ formaldehyde for $1 \mathrm{~h}$ at $4{ }^{\circ} \mathrm{C}$ and then permeabilized with $0.1 \%$ triton in blocking buffer (0.5\% saponin in PBS, $\mathrm{pH} 7.4$, plus $10 \%$ sheep serum) for $30 \mathrm{~min}$. Oocytes were then washed free of triton and continuously blocked for another $90 \mathrm{~min}$ at room temperature. Oocytes were incubated overnight at $4{ }^{\circ} \mathrm{C}$ with primary antibody (rabbit anti-phospho-AMPKalpha-PT172, 1:100; Cell Signaling) and then washed in blocking buffer and incubated with FITC-conjugated sheep anti-rabbit antibody (1:1000; Cell Signaling) at room temperature for $1 \mathrm{~h}$. Oocytes were then washed again and mounted on slides with Vectashield containing DAPI to stain chromatin. Images were viewed on a laser scanning confocal microscope
(Carl Zeiss Co., Thornwood, NY, USA) with a $40 \times$ objective. Digitally recorded images were exported by a LSM Examiner (Carl Zeiss Co).

\section{Statistical analysis}

Oocyte maturation experiments were repeated at least three times with at least 25 oocytes per group per experiment. Data are reported as mean percentage GVB \pm s.E.M. Maturation frequencies were analyzed statistically by ANOVA followed by Duncan's multiple range test, or paired comparisons were analyzed by Student's $t$-test. For all statistical analyses, a $P$ value $<0.05$ was considered significant.

\section{Declaration of interest}

There is no conflict of interest that could be perceived as prejudicing the impartiality of the research reported.

\section{Funding}

This work was supported by a grant from the NIH (R01 40392) to S M D.

\section{References}

Bos JL 2006 Epac proteins: multi-purpose cAMP targets. Trends in Biochemical Sciences 31 680-686.

Chen J \& Downs SM 2008 AMP-activated protein kinase is involved in hormone-induced mouse oocyte meiotic maturation in vitro. Developmental Biology 313 47-57.

Chen J, Hudson E, Chi MM, Chang AS, Moley KH, Hardie DG \& Downs SM 2006 AMPK regulation of mouse oocyte meiotic resumption in vitro. Developmental Biology 29 227-238.

Conti M, Andersen CB, Richard F, Mehats C, Chun S-Y, Horner K, Jin C \& Tsafriri A 2002 Role of cyclic nucleotide signaling in oocyte maturation. Molecular and Cellular Endocrinology 187 153-159.

Davies SP, Carling D \& Hardie DG 1989 Tissue distribution of the AMPactivated protein kinase, and lack of activation by cyclic-AMPdependent protein kinase, studied using a specific and sensitive peptide assay. European Journal of Biochemistry 186 123-128.

Davies SP, Helps NR, Cohen PTW \& Hardie DG 1995 5'-AMP inhibits dephosphorylation, as well as promoting phosphorylation, of the AMPactivated protein kinase. Studies using bacterially expressed human protein phosphatase- $2 \mathrm{C}(\alpha)$ and native bovine protein phosphatase-2Ac. FEBS Letters 377 421-425.

Dekel N 1988 Regulation of oocyte maturation. The role of cAMP. Annals of the New York Academy of Sciences 541 211-216.

Dekel N, Lawrence TS, Gilula NB \& Beers WH 1981 Modulation of cellto-cell communication in the cumulus-oocyte complex and the regulation of oocyte maturation by LH. Developmental Biology $\mathbf{8 6}$ 356-362.

Dekel N, Aberdam E \& Sherizly I 1984 Spontaneous maturation in vitro of cumulus-enclosed rat oocytes is inhibited by forskolin. Biology of Reproduction 31 244-250.

Dostmann WR, Taylor SS, Genieser HG, Jastorff B, Doskeland SO \& Ogreid D 1990 Probing the cyclic nucleotide binding sites of cAMPdependent protein kinases I and II with analogs of adenosine $3^{\prime}, 5^{\prime}$-cyclic phosphorothioates. Journal of Biological Chemistry 265 10484-10491.

Downs SM, Coleman DL, Ward-Bailey PF \& Eppig JJ 1985 Hypoxanthine is the principal inhibitor of murine oocyte maturation in a low molecular weight fraction of porcine follicular fluid. PNAS 82 454-458. 
Downs SM, Daniel SA \& Eppig JJ 1988 Induction of maturation in cumulus cell-enclosed mouse oocytes by follicle-stimulating hormone and epidermal growth factor: evidence for a positive stimulus of somatic cell origin. Journal of Experimental Zoology 245 86-96.

Downs SM, Daniel SAJ, Bornslaeger EA, Hoppe PC \& Eppig JJ 1989 Maintenance of meiotic arrest in mouse oocytes by purines: modulation of cAMP levels and cAMP phosphodiesterase activity. Gamete Research 23 323-334.

Edry I, Sela-Abramovich S \& Dekel N 2006 Meiotic arrest of oocytes depends on cell-to-cell communication in the ovarian follicle. Molecular and Cellular Endocrinology 252 102-106.

Enserink JM, Christensen AE, de Rooij J, van Triest $M$, Schwede F, Genieser HG, Doskeland SO, Blank JL \& Bos JL 2002 A novel Epacspecific cAMP analogue demonstrates independent regulation of Rap1 and ERK. Nature Cell Biology 4 901-906.

Eppig JJ, Ward-Bailey PF \& Coleman DL 1985 Hypoxanthine and adenosine in murine ovarian follicular fluid: concentrations and activity in maintaining oocyte meiotic arrest. Biology of Reproduction 33 1041-1049.

Freudzon L, Norris RP, Hand AR, Tanaka S, Saeki Y, Jones TLZ, Rasenick MM, Berlot CH, Mehlmann LM \& Jaffe LA 2005 Regulation of meiotic prophase arrest in mouse oocytes by GPR3, a constitutive activator of the Gs G protein. Journal of Cell Biology 171 255-265.

Ha J, Daniel S, Broyles SS \& Kim KH 1994 Critical phosphorylation sites for acetyl-CoA carboxylase activity. Journal of Biological Chemistry 269 22162-22168.

Han SJ, Vaccari S, Nedachi T, Andersen CB, Kovacina KS, Roth RA \& Conti M 2006 Protein kinase B/Akt phosphorylation of PDE3A and its role in mammalian oocyte maturation. EMBO Journal 25 5716-5725.

Hardie DG \& Hawley SA 2001 AMP-activated protein kinase: the energy charge hypothesis revisited. BioEssays 23 1112-1119.

Hashimoto N, Kishimoto T \& Nagahama Y 1985 Induction and inhibition of meiotic maturation in follicle-enclosed mouse oocytes by forskolin. Development, Growth \& Differentiation 27 709-716.

Hawley SA, Gadalla AE, Olsen GS \& Hardie DG 2002 The antidiabetic drug metformin activates the AMP-activated protein kinase cascade via an adenine nucleotide-independent mechanism. Diabetes 51 2420-2425.

Haystead TA, Moore F, Cohen P \& Hardie DG 1990 Roles of the AMPactivated and cyclic-AMP-dependent protein kinases in the adrenalineinduced inactivation of acetyl-CoA carboxylase in rat adipocytes. European Journal of Biochemistry 187 199-205.

Henin N, Vincent MF \& Van den Berghe G 1996 Stimulation of rat liver AMP-activated protein kinase by AMP analogues. Biochimica et Biophysica Acta 1290 197-203.

Horner K, Livera G, Hinckley M, Trinh K, Storm D \& Conti M 2003 Rodent oocytes express an active adenylyl cyclase required for meiotic arrest. Developmental Biology 258 385-396.

Hubbard CJ 1985 The effects of forskolin and LH on cAMP changes and maturation in the follicle-enclosed oocytes of hamsters. Acta Endocrinologica 110 413-420.

Hubbard CJ 1986 Cyclic AMP changes in the component cells of Graafian follicles: possible influences on maturation in the follicle-enclosed oocytes of hamsters. Developmental Biology 118 343-351.

Hurley RL, Barre LK, Wood SD, Anderson KA, Kemp BE, Means AR \& Witters LA 2006 Regulation of AMP-activated protein kinase by multisite phosphorylation in response to agents that elevate cellular CAMP. Journal of Biological Chemistry 281 36662-36672.

Kalinowski RR, Berlot CH, Jones TLZ, Ross LF, Jaffe LA \& Mehlmann LM 2004 Maintenance of meiotic prophase arrest in vertebrate oocytes by a Gs protein-mediated pathway. Developmental Biology 267 1-13.

LaRosa C \& Downs SM 2006 Stress stimulates AMP-activated protein kinase and meiotic resumption in mouse oocytes. Biology of Reproduction 74 585-592.

Masciarelli S, Horner K, Liu C, Park SH, Hinckley M, Hockman S, Nedachi T, Jin C, Conti M \& Manganiello V 2004 Cyclic nucleotide phosphodiesterase 3A-deficient mice as a model of female infertility. Journal of Clinical Investigation 114 196-205.

Mehlmann LMJ, Teresa LZ \& Jaffe LA 2002 Meiotic arrest in the mouse follicle maintained by a Gs protein in the oocyte. Science 297 1343-1345.
Mehlmann LM, Saeki Y, Tanaka S, Brennan TJ, Evsikov AV, Pendola FL, Knowles BB, Eppig JJ \& Jaffe LA 2004 The Gs-linked receptor GPR3 maintains meiotic arrest in mammalian oocytes. Science 306 1947-1950.

Moor RM \& Heslop JP 1981 Cyclic AMP in mammalian follicle cells and oocytes during maturation. Journal of Experimental Zoology 216 205-209.

Ratchford AM, Chang AS, Chi MM, Sheridan R \& Moley KH 2007 Maternal diabetes adversely affects AMP-regulated protein kinase activity and cellular metabolism in murine oocytes. American Journal of Physiology. Endocrinology and Metabolism 293 E1198-E1206.

Richard FJ, Tsafriri A \& Conti M 2001 Role of phosphodiesterase type 3A in rat oocyte maturation. Biology of Reproduction 65 1444-1451.

de Rooij J, Zwartkruis FJT, Verheijen MHG, Cool RH, Nijman SMB, Wittinghofer A \& Bos JL 1998 Epac is a Rap1 guanine-nucleotideexchange factor directly activated by cyclic AMP. Nature 396 474-477.

Ruderman NB, Park H, Kaushik VK, Dean D, Constant S \& Saha AK 2003 AMPK as a metabolic switch in rat muscle, liver and adipose tissue after exercise. Acta Physiologica Scandinavica 178 435-442.

Salustri A, Petrungaro S, De Felici M, Conti M \& Siracusa G 1985 Effect of follicle-stimulating hormone on cyclic adenosine monophosphate level and on meiotic maturation in mouse cumulus cell-enclosed oocytes cultured in vitro. Biology of Reproduction 33 797-802.

Sandberg M, Butt E, Nolte C, Fischer L, Halbrugge M, Beltman J, Jahnsen T, Genieser HG, Jastorff B \& Walter U 1991 Characterization of Sp-5,6dichloro-1-beta-D-ribofuranosylbenzimidazole- $\quad 3$ ',5'-monophosphorothioate (Sp-5,6-DCl-cBiMPS) as a potent and specific activator of cyclic-AMP-dependent protein kinase in cell extracts and intact cells. Biochemical Journal 279 521-527.

Sanders MJ, Grondin PO, Hegarty BD, Snowden MA \& Carling D 2007 Investigating the mechanism for AMP activation of the AMP-activated protein kinase cascade. Biochemical Journal 403 139-148.

Schultz RM, Montgomery RR \& Belanoff JR 1983 Regulation of mouse oocyte meiotic maturation: implication of a decrease in oocyte cAMP and protein dephosphorylation in commitment to resume meiosis. Developmental Biology 97 264-273.

Shitsukawa K, Andersen CB, Richard FJ, Horner AK, Wiersma A, van Duin M \& Conti M 2001 Cloning and characterization of the cyclic guanosine monophosphate-inhibited phosphodiesterase PDE3A expressed in mouse oocyte. Biology of Reproduction 65 188-196.

Urner F, Herrmann WL, Baulieu EE \& Schorderet-Slatkine S 1983 Inhibition of denuded mouse oocyte meiotic maturation by forskolin, an activator of adenylate cyclase. Endocrinology 113 1170-1172.

Webb RJ, Marshall F, Swann K \& Carroll J 2002 Follicle-stimulating hormone induces a gap junction-dependent dynamic change in (cAMP) and protein kinase A in mammalian oocytes. Developmental Biology 246 441-454.

Wiersma A, Hirsch B, Tsafriri A, Hanssen RGJM, Van de Kant M, Kloosterboer HJ, Conti M \& Hsueh AJW 1998 Phosphodiesterase 3 inhibitors suppress oocyte maturation and consequent pregnancy without affecting ovulation and cyclicity in rodents. Journal of Clinical Investigation 102 532-537.

Yoshimura Y, Nakamura Y, Ando M, Jinno M, Oda T, Karube M, Koyama N \& Nanno T 1992a Stimulatory role of cyclic adenosine monophosphate as a mediator of meiotic resumption in rabbit oocytes. Endocrinology 131 351-356.

Yoshimura Y, Nakamura Y, Oda T, Ando M, Ubukata Y, Karube M, Koyama N \& Yamada H 1992b Induction of meiotic maturation of follicle-enclosed oocytes of rabbits by a transient increase followed by an abrupt decrease in cyclic AMP concentration. Journal of Reproduction and Fertility 95 803-812.

Zhou G, Myers R, Li Y, Chen Y, Shen X, Fenyk-Melody J, Wu M, Ventre J, Doebber T, Fujii $\mathbf{N}$ et al. 2001 Role of AMP-activated protein kinase in mechanism of metformin action. Journal of Clinical Investigation 108 1167-1174.

Received 1 January 2009

First decision 18 February 2009

Revised manuscript received 13 July 2009

Accepted 21 August 2009 